\title{
BMJ Open Combination of InnoSEAL plus TR band compared with TR band alone for radial artery outcomes in patients undergoing transradial coronary intervention (InnoSEAL-II): an open- label randomised controlled trial (protocol)
}

\author{
Saba Aijaz, ${ }^{1}$ Sana Sheikh (1) ,2 Asad Pathan ${ }^{1}$
}

To cite: Aijaz S, Sheikh S, Pathan A. Combination of InnoSEAL plus TR band compared with TR band alone for radial artery outcomes in patients undergoing transradial coronary intervention (InnoSEAL-II): an open-label randomised controlled trial (protocol). BMJ Open 2020;10:e042101. doi:10.1136/ bmjopen-2020-042101

- Prepublication history and supplemental material for this paper is available online. To view these files, please visit the journal online (http://dx.doi. org/10.1136/bmjopen-2020042101).

Received 10 July 2020 Revised 17 November 2020 Accepted 25 November 2020

Check for updates

(c) Author(s) (or their employer(s)) 2020. Re-use permitted under CC BY-NC. No commercial re-use. See rights and permissions. Published by BMJ.

${ }^{1}$ Department of Cardiology, Tabba Heart Institute, Karachi, Sindh, Pakistan

${ }^{2}$ Clinical Research Department, Tabba Heart Institute, Karachi, Sindh, Pakistan

Correspondence to

Dr Asad Pathan;

asadzpathan@gmail.com

\section{ABSTRACT}

Introduction About $2 \%-30 \%$ of cardiac catheterisation procedures get complicated by radial artery occlusion (RA0). Ensuring patent haemostasis appears to be an important factor in reducing RA0. Currently employed method is a radial compression device (RCD) such as transradial band (TRB) that take hours to achieve haemostasis and cause discomfort to the patients. Haemostatic pads offer an alternative to RCD with reduced time to achieve haemostasis. Our trial aims to determine the non-inferiority of the catecholamine chitosan-based pad (InnoSEAL haemostatic pad) used in conjunction with TRB (InnoSEAL +TRB) when compared with the TRB alone in reducing composite adverse access site outcomes. Methods and analysis It will be an open-label, parallel, randomised controlled trial on 714 adult patients (325 in each arm) undergoing coronary procedure using transradial approach at a cardiac health facility over 7 months duration. InnoSEAL patch along with TRB will be used to control bleeding in intervention arm and TRB alone in control arm, which is the standard practice. Study primary outcomes include RAO and haematoma; secondary outcomes are compression time, patient discomfort, time to discharge and ease of use of the intervention technique by the healthcare staff. $\chi^{2}$ test will be used to compare the categorical outcomes between two arms and student's t-test for continuous outcomes. A $p$ value of $<0.05$ will be considered significant.

Ethics and dissemination Ethical approval for the study has been obtained from the Institutional Review Board of Tabba Heart Institute number IORG0007863. Findings will be disseminated through seminars and scientific publications.

Trial registration number NCT04380883; Pre-results.

\section{INTRODUCTION}

Transradial procedures are frequently complicated by radial artery occlusion (RAO) with a reported incidence of $2 \%-30 \%$. $^{1}$ RAO may
Strengths and limitations of this study

- This is the first powered clinical trial comparing chitosan-based haemostatic pad to the pneumatic band. The trials done previously were on a lesser number of patients with no power calculation.

- One of the secondary outcomes of the study is the ease of the use of any of the techniques of access site closure by the cath lab staff. This will inform the feasibility of translating the trial results into practice.

- The lack of blinding of the data collectors is a limitation of the study.

limit future use of this site for catheterisation, as an arterial conduit for surgical revascularisation or for haemodialysis access. Independent predictors of RAO are the diameter of the sheath and its relationship to the size of the radial artery, amount of heparin used, post-procedure compression time and presence of anterograde flow in the artery during haemostasis. ${ }^{2}$ Ensuring patent haemostatic compression, that is, the control of arterial bleeding while maintaining radial arterial flow appears to be an important factor in reducing RAO. ${ }^{3}$

Haemostasis after radial sheath removal has been achieved using multiple methods including manual compression, tourniquets and compression bandages, a variety of pneumatic compression devices, and more recently haemostatic pads impregnated with procoagulant chemicals. Currently, the most frequently employed method for haemostasis following transradial procedures is a radial compression device (RCD). ${ }^{4}$ 
Haemostatic pads offer an alternative to RCD, where overall compression time is inherently low and patent haemostasis can possibly be achieved. Chitosan, one of the materials used in haemostasis pads, is a biodegradable polysaccharide, which is converted to a haemostatic agent after contact with blood, and has shown similar efficacy in terms of reducing local bleeding and haematoma formation compared with RCDs with the added advantage of the shorter time of compression and therefore less patient discomfort. ${ }^{5}$

The combined use of transradial band (TRB) with a haemostatic device may allow ease of use with reduced haemostasis time. ${ }^{6}$ Patent haemostasis is also likely to be achieved with the combined use of both devices and possibly improve radial artery patency with the added benefit of reduced bleeding complications. So far two trials have been undertaken that compared RCD alone with RCD plus catechol conjugated homeostasis pads. ${ }^{78}$ Both trials showed the reduced time to achieve haemostasis with RCD plus haemostatic pad but both were underpowered with a small number of participants. Also, we did not find any trial from lower middle-income countries (LMICs), hence we planned a trial with adequate power, which will test the applicability of the haemostatic pad with the pneumatic band in an LMIC.

Our trial aims to test the hypothesis that compared with TRB alone, catecholamine chitosan-based pad (InnoSEAL haemostatic pad, InnoTherapy, S Korea) used in conjunction with TRB (InnoSEAL +TRB) is non-inferior in terms of the composite adverse access site outcomes, that is, RAO within 24 hours and haematoma as per Bleeding Academic Research Consortium grading. ${ }^{9}$ Secondary objectives include testing the non-inferiority of InnoSEAL + TRB in terms of ease of use by the cath lab staff compared with TRB; and superiority of InnoSEAL +TRB for shortened total haemostasis time, total observation time for the radial site and time to hospital discharge for daycare patients' subgroup. Finally, we aim to test the superiority of InnoSEAL +TRB for reduced patient discomfort compared with TRB alone.

\section{METHODS AND ANALYSIS}

This open-label randomised controlled trial will be conducted in the cardiac catheterisation laboratory (CCL) at Tabba Heart Institute (THI) Karachi, Pakistan. THI is a 160 bedded cardiac tertiary care hospital with 24 hours emergency and facilities for primary percutaneous coronary intervention (PCI) and cardiac surgeries. The institution has two fully equipped CCL. There is a fully trained and experienced faculty of more than 15 cardiologists and 06 interventionists. On average, more than 1400 PCIs are performed annually. This is one of the major referral centres in the city for primary PCI. THI is also a teaching hospital with approved training programmes in adult cardiology and interventional cardiology.
Adult patients of both gender undergoing coronary procedure using transradial approach including diagnostic coronary angiogram (CAG) or PCI and who are haemodynamically stable will be included. Patients in whom radial sheath of larger than $6 \mathrm{~F}$ will be used; who are on continuous infusion of anticoagulants (unfractionated heparin, gpIIb/IIIa inhibitors) after the procedure or ongoing anticoagulation therapy (warfarin, rivaroxaban) or with international normalised ratio (INR) $>3$ will be excluded. Also, patients who are diagnosed with ipsilateral arteriovenous fistula, Barbeau's class $\mathrm{D}$, or have a history of RAO at baseline or unable to give consent will be excluded.

All consecutive patients coming to CCL for coronary procedures will be screened for eligibility by data collectors. If the participants meet the eligibility criteria of the study, they will be offered to participate in the trial. Before the start of the coronary procedure, patients will be approached for enrolment in the study and informed consent will be taken. If the participant assigns a surrogate, then consent will be signed by the surrogate. In case of urgent need of coronary procedures, consent will be taken post coronary procedure when the patient is shifted in the cath lab recovery area. The consent form will be provided in English or Urdu according to the patient's preference. One signed copy will be given to the patient for the reference. For an uneducated patient, the form will be verbally read and thoroughly explained by the research coordinator. A thumb impression will be obtained along with the sign from a witness.

Some participants might be excluded after giving consent due to procedure-related exclusion criteria like continued intravenous anticoagulation as per the discretion of the primary interventionist. To preserve randomisation and to reduce utilisation of the resources, the allocation of treatment will be performed at the end of the procedure.

Randomisation will be performed by a core research team member of the hospital who is not part of this study using variable-sized blocks of 4 or 6 through computer software and will be kept in password-protected computer. Randomisation will be revealed to the data collector over the phone by the same person who generated the sequence. Blinding of the participant or the data collectors is not possible in this study.

Data collectors will receive half-day training for consent taking and data collection. A dry run of the study will be undertaken to identify challenges in the study execution and provide hands-on training to the research staff. The plan is to recruit about 10 participants during the dry run. The data of these patients will not be used in the final analysis.

Procedure details: All patients receive intravenous heparin at a dose of at least $70-100 \mathrm{IU} / \mathrm{kg}$ at the start of the procedure for diagnostic CAG; the dose will be higher for PCI at the discretion of the primary operators. After insertion of radial sheath, all patients receive intraradial verapamil (maximum of $5 \mathrm{mg}$ ) and 


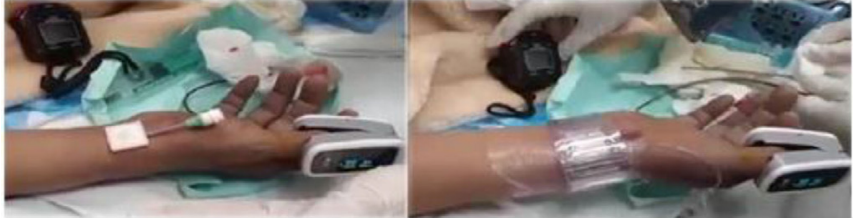

Figure 1 InnoSEAL patch is applied over the sheath and transradial band is tied over it.

nitroglycerin (maximum $500 \mathrm{mg}$ ) at a dose titrated to the patient's blood pressure. At the end of the radial procedures, both the groups will receive up to $500 \mathrm{mg}$ of nitroglycerin into the sheath according to patient's blood pressures.

\section{Intervention group (InnoSEAL+TRB)}

1. The sheath is pulled $2-3 \mathrm{~cm}$ and the area surrounding the puncture site is cleaned and dried.

2. The InnoSEAL is placed over the sheath at the puncture site.

3. A transparent adhesive clear dressing is placed over the InnoSEAL.

4. TR band is applied over the transparent adhesive clear dressing centred over the InnoSEAL.

5. $12 \mathrm{cc}$ air is inflated to apply pressure (figure 1).

6 . The sheath is removed. There is a small amount of blood at the sheath tip that is enough to activate the haemostatic pad.

7. Air is removed from the TR band at 20 min intervals, removing $2 \mathrm{cc}$ air at $20 \mathrm{~min}, 4 \mathrm{cc}$ at $40 \mathrm{~min}$ and $6 \mathrm{cc}$ at $60 \mathrm{~min}$ from the time of sheath removal. Thus, 10 cc air remains at $20 \mathrm{~min}, 6 \mathrm{cc}$ at $40 \mathrm{~min}$ and $0 \mathrm{cc}$ (total deflation) at $60 \mathrm{~min}$. Total compression time is recorded.

8. TRB is left in place with $0 \mathrm{cc}$ air for further $30 \mathrm{~min}$ and the patient will keep his/her arm in resting position. The total duration of TRB application including the armrest is $90 \mathrm{~min}$. Reverse Barbeau's test is repeated just after the removal of TRB.

9. If there is bleeding at any time, 2 cc air is reintroduced in the TRB.

10. After the band is removed, clear adhesive dressing and InnoSEAL is left in place.

11. Radial site is observed for rebleeding for another 30 min after removal of the TRB.

12. Adhesive dressing and InnoSEAL is removed after 24 hours of the procedure. If the patient remains admitted, the site is reassessed at 24 hours for RAO and haematoma. For patients discharging on the same day, final observation for RAO and haematoma will be made at the time of TR band removal after leaving it at zero pressure for $30 \mathrm{~min}$. Same day patients will be discharged 1 hour after the end of the observation period.

13. Patients screened positive for RAO will be reassessed at 6 months.

\section{Standard group (TRB alone)}

(Protocol is identical for both diagnostic angiogram and PCI)

1. The area surrounding the puncture site is cleaned and dried.

2. The sheath is pulled out $2-3 \mathrm{~cm}$.

3. TRB is applied centred over the puncture site and the bladder is inflated with $15 \mathrm{cc}$ air to apply pressure.

4. The sheath is now removed.

5. Air is removed gradually leaving at least $10 \mathrm{cc}$ air provided, there is no oozing. In case of oozing at any time, $2 \mathrm{cc}$ air is reintroduced and the final amount of air is recorded. This time is recorded as time of start of haemostasis protocol.

6. At $90 \mathrm{~min}$ from the time of haemostasis protocol, $2 \mathrm{cc}$ air is removed. From then on at every 20 min interval, further air is removed starting from with maximum $4 \mathrm{cc}$ from the remaining air at first $20 \mathrm{~min}(110 \mathrm{~min}$ from the start of the protocol), followed by maximum $6 \mathrm{cc}$ at $40 \mathrm{~min}$ and then if still there is remaining air, maximum $6 \mathrm{cc}$ at every $20 \mathrm{~min}$ until all the air is removed from the bladder. Total compression time is recorded.

7. If there is bleeding at any time, 2 cc air is reintroduced in the TRB.

8. TRB is left in place at $0 \mathrm{cc}$ air for further $30 \mathrm{~min}$ for safety and Barbeau's test is repeated just after TRB removal.

9. Radial site is further observed for rebleeding, for another $30 \mathrm{~min}$ after the removal of the TRB.

10. If the patient remains admitted, the site is reassessed at 24 hours for RAO and haematoma. For patients discharging on the same day, final observation for RAO and haematoma will be made at the time of TRB removal after leaving it at zero pressure for $30 \mathrm{~min}$.

11. Same day patients will be discharged 1 hour after the end of the observation period.

12. Patients screened positive for RAO will be reassessed at 6 months.

\section{Patient and public involvement}

No patient involved.

\section{Sample size calculation}

As per our standard practice of only TRB-based haemostasis, the radial occlusion rate is $9.4 \%$ and radial haematoma rate is $3.4 \%$ (combined event rate of $12.8 \%$ ). Through a pilot on 40 patients to devise the protocol for InnoSEAL +TRB, we determined a combined rate of $17.7 \%$ (RAO: $7.7 \%$, haematoma: $10 \%$ ). If there is a true difference in favour of the experimental treatment of $4.89 \%$ (17.7\% vs $12.8 \%)$, then a total of 648 patients (324 in each group) are required to be $80 \%$ sure that the upper limit of a one-sided $97.5 \%$ CI (or equivalently a 95\% two-sided CI) will exclude a difference in favour of the standard group of more than $3 \%$. If $10 \%$ attrition is considered, then 33 additional patients are required in 
each group, thus 357 patients in each group and a total of 714 patients.

\section{Study outcome assessment}

Primary outcome

It is a combination of RAO and presence of haematoma of any grade.

1. RAO: radial artery will be defined as occluded if reverse Barbeau's test shows absence of flow on pulse oximetry just after the removal of the TRB in both groups. In patients identified to have RAO, findings will be confirmed using US Duplex using colour flow with pulse wave imaging within 24 hours of the radial procedure. US Duplex will be performed during the index hospital stay.

2. Radial haematoma: radial artery site will be assessed for presence of haematoma at the end of haemostasis protocol. Haematoma will be marked if present, and graded according to categories of I-IV. ${ }^{10}$ Haematomas grade II-IV will be considered significant.

Patients who develop these complications will be managed as per the standard of care in the hospital. For RAO, usually no intervention is required. Radial haematoma is also a self-limiting complication and occasionally may need manual compression and milking and use of sphygmomanometer cuff. After 24 hours of radial haemostasis, no patient follow-up is involved in the study.

\section{Secondary outcomes}

These include:

1. Ease of use: ease of use of InnoSEAL will be assessed by a system usability scale, ${ }^{11} 5$-point Likert scale by the cath lab personnel who routinely perform postprocedure haemostasis. Ease of use is reported as percentage acceptability, where $100 \%$ means highly acceptable and vice versa. Ease of use proforma will be filled by the participating cath lab staffs at the end of the study. Informed consent will be taken from the cath lab staff.

2. Total compression time: time required from radial sheath removal to the removal of all the air from TRB.

3. Total observation time: time required from radial sheath removal to the removal of TRB (included time for observation at $0 \mathrm{cc}$ for safety purpose).

4. Time to hospital discharge: (for daycare patients only) time from removal of radial sheath till patient discharge from hospital.

5. Patient discomfort: standard visual pain scale of 1-10 will be used. ${ }^{12}$ Pain will be assessed after the haemostasis protocol is ended.

\section{Adverse events}

Rebleeding at the end of intervention protocol in either group, that is, bleeding after removal of InnoSEAL $+\mathrm{TRB}$ or TRB will be considered as an adverse event. If adverse event occurred, it will be recorded and TRB will be applied at the puncture site and inflated with $2 \mathrm{cc}$ air or more till no bleeding is observed. Then $2 \mathrm{cc}$ air will be removed at $20 \mathrm{~min}, 4 \mathrm{cc}$ at $40 \mathrm{~min}$ and $6 \mathrm{cc}$ at $60 \mathrm{~min}$ when needed. After 30 min of 0 air, TRB will be removed and site will be observed for bleeding. Final observation will be made after $30 \mathrm{~min}$ of removal of TRB. IRB and funders will be informed about the adverse events in the final report.

\section{Statistical analysis plan}

Data quality will be assured by random checking of $10 \%$ of the weekly data by the study investigators and random observation of the participant recruitment and live data collection. Data will be entered in Microsoft Access, which will have in-built checks (like value ranges and pop-up for invalid values) to minimise data entry errors. Means and SD will be reported for continuous data based on normality assumption along with histograms and compared using independent student's t-test. And frequencies with percentages will be reported for the categorical variables. The differences between categorical variables will be examined by the $\chi^{2}$ test and $p$ value $<0.05$ will be considered significant. Statistical analysis will be performed using the SPSS V.24.0. For comparison of baseline characteristics, independent student's t-test and $\chi^{2}$ tests will be used for quantitative and categorical variables, respectively. $\chi^{2}$ test will be used to assess primary composite outcome and its components. Independent sample t-test will be used for comparison of time intervals. Kruskall Wallis test will be used for significance testing between ordinal secondary outcomes of ease of use and patient discomfort. Intention to treat analysis will be undertaken. If there would be many cross-overs, then per protocol analysis will also be conducted and results of both will be compared and discussed.

\section{ETHICS AND DISSEMINATION}

Ethical approval will be taken from Institutional Review Board of THI. Written informed consent will be obtained from all study participants prior to the enrolment in the study (online supplemental appendix: consent form).

No personal or clinical information of study participants will be made public. All study information will be stored in lock and key and in password-protected software after data entry. The study will be carried out in compliance with Good Clinical Practice guidelines and all the study investigators and key members will be IRB certified to conduct human research. IRB will conduct an independent audit of the trial execution as per their policy.

Study findings will be disseminated to the study hospital staff and intervention arm with favourable results will be implemented as hospital protocol for radial site closure after cath lab procedures. The results will also be published in scientific journals and will be presented in seminar and conferences.

Contributors SA developed the protocol, estimated sample size and data analysis plan, AP initially conceived the idea and provided intellectual input to design the methodology and critically reviewed the protocol. SS drafted the protocol for publication, is involved in the execution of the trial, as well as the supervision of the study staff. 
Funding This work was supported by InnoTherapy $\mathrm{S}$ grant number THIINNOSEAL2-2020, Korea. Funding agency has no role in designing the study, writing the protocol, study execution, data management or analysis and interpretation of the results. Sponsor contact number: 201-944-0445.

Competing interests None declared.

Patient consent for publication Not required.

Provenance and peer review Not commissioned; externally peer reviewed.

Supplemental material This content has been supplied by the author(s). It has not been vetted by BMJ Publishing Group Limited (BMJ) and may not have been peer-reviewed. Any opinions or recommendations discussed are solely those of the author(s) and are not endorsed by BMJ. BMJ disclaims all liability and responsibility arising from any reliance placed on the content. Where the content includes any translated material, BMJ does not warrant the accuracy and reliability of the translations (including but not limited to local regulations, clinical guidelines, terminology, drug names and drug dosages), and is not responsible for any error and/or omissions arising from translation and adaptation or otherwise.

Open access This is an open access article distributed in accordance with the Creative Commons Attribution Non Commercial (CC BY-NC 4.0) license, which permits others to distribute, remix, adapt, build upon this work non-commercially, and license their derivative works on different terms, provided the original work is properly cited, appropriate credit is given, any changes made indicated, and the use is non-commercial. See: http://creativecommons.org/licenses/by-nc/4.0/.

ORCID iD

Sana Sheikh http://orcid.org/0000-0001-7981-2957

\section{REFERENCES}

1 Rashid M, Kwok CS, Pancholy S, et al. Radial artery occlusion after Transradial interventions: a systematic review and meta-analysis. $J$ Am Heart Assoc 2016;5:e002686.
2 Mamas MA, Fraser DG, Ratib K, et al. Minimising radial injury: prevention is better than cure. Eurolntervention 2014;10:824-32.

3 Pancholy S, Coppola J, Patel T, et al. Prevention of radial artery occlusion-patent hemostasis evaluation trial (PROPHET study): a randomized comparison of traditional versus patency documented hemostasis after transradial catheterization. Catheter Cardiovasc Interv 2008;72:335-40.

4 Shroff AR, Fernandez C, Vidovich MI, et al. Contemporary transradial access practices: results of the second international survey. Catheter Cardiovasc Interv 2019;93:1276-87.

5 Kumar DS, Lei H, Wenliang C, et al. A novel role of chitosan in reducing vascular access bleeding complications after transradial angiography. China Medical Abstracts 2011;20:57-60.

6 Seto AH, Rollefson W, Patel MP, et al. Radial haemostasis is facilitated with a potassium ferrate haemostatic patch: the Statseal with TR band assessment trial (STAT). Eurolntervention 2018;14:e1236-42.

7 Kang S-H, Han D, Kim S, et al. Hemostasis pad combined with compression device after transradial coronary procedures: a randomized controlled trial. PLoS One 2017;12:e0181099.

8 Roberts JS, Niu J, Pastor-Cervantes JA. Comparison of Hemostasis Times with a Chitosan-Based Hemostatic Pad (Clo-SurPlus Radial $^{\mathrm{TM}}$ ) vs Mechanical Compression (TR Band $囚$ ) Following Transradial Access: A pilot Study. Cardiovasc Revasc Med 2019;20:871-4.

9 Mehran R, Rao SV, Bhatt DL, et al. Standardized bleeding definitions for cardiovascular clinical trials: a consensus report from the bleeding academic research Consortium. Circulation 2011;123:2736-47.

10 Shroff A, Siddiqui S, Burg A, et al. Identification and management of complications of transradial procedures. Curr Cardiol Rep 2013;15:350.

11 Bangor A, Kortum PT, Miller JT. An empirical evaluation of the system usability scale. Int J Hum Comput Interact 2008;24:574-94.

12 McCormack HM, Horne DJ, Sheather S. Clinical applications of visual analogue scales: a critical review. Psychol Med 1988;18:1007-19. 